“This work has been submitted to the IEEE for possible publication. Copyright may be transferred without notice, after which this version may no longer be accessible."

\title{
Development of Ingestible Thermometer with Built-in Coil Antenna Charged by Gastric Acid Battery and Demonstration of Long-Time in vivo Telemetry
}

\author{
*Shinya Yoshida, Hiroshi Miyaguchi, and Tsutomu Nakamura
}

\begin{abstract}
Objective: A safe and affordable ingestible thermometer measuring core body temperature has the potential to become a future healthcare device for versatile applications in daily life. In this study, we developed an ingestible thermometer charged by a gastric acid battery. Methods: The device can operate in bowels by using the charged energy in multilayer ceramic capacitors as a storage capacitor. Adopting this strategy for energy storage solves the issues related to a conventional button battery: risk of injury to the digestive tract, bad disposability, and degradation. Additionally, to make it easy to assemble a coil antenna and electrical circuits in the device automatically, we developed a fabrication process based on a vertical stacking process of printed circuit boards with coil patterns. The dimensions of the prototyped device were smaller than those of existing ingestible thermometers. Results: In an experiment involving a dog, we successfully recorded the temperature in the digestive tract for $24 \mathrm{~h}$ in cycles of approximately 10 or $20 \mathrm{~min}$, using a rectal thermometer and an existing ingestible thermometer as references. The temperature variations in time among our device, rectal thermometer, and existing ingestible thermometer were almost parallel. Conclusion: The recording ability of the core body temperature using our device has the potential to measure basal body temperature during sleep, the circadian rhythm, and fever type easily and robustly in daily life. Significance: Our ingestible thermometer is a step toward the development of sensors that can be swallowed for preventive medicine and health promotion.
\end{abstract}

Index Terms-Biomedical telemetry, Core body temperature sensors, System integration, Ingestible, swallowable, electronic pill

\section{INTRODUCTION}

Vital sensing or monitoring in daily life has prospects in promoting health and detecting diseases at an early stage. The general vision is referred to as "digital heath," "mobile health," "Internet of Medical Things," etc., and it is gradually becoming established among many people because of advances in

This work was supported in part by JST COI, Grant Number JPMJCE1303, and in part by the TERUMO Life Science Foundation.

S. Yoshida is with Graduate School of Engineering, Tohoku University, Sendai 980-8579, Japan (correspondence e-mail: s-yoshida@ tohoku.ac.jp). H. Miyaguchi is with the Micro System Integration Center, Tohoku University, Sendai 980-8579, Japan. T. Nakamura is with the Promotion Office of Strategic Innovation, Tohoku University, Sendai 980-8579, Japan.

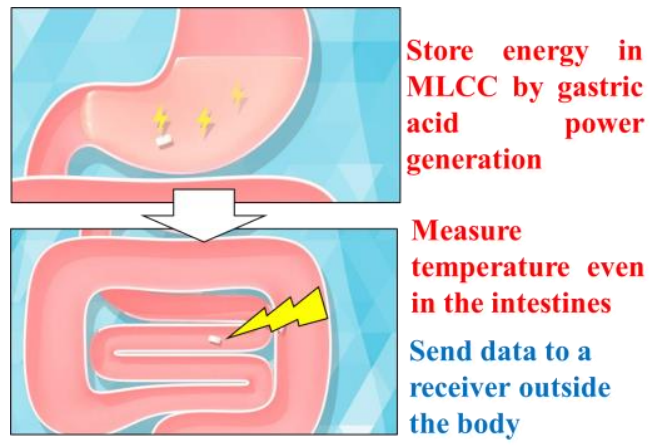

Fig. 1. Schematic illustration of the ingestible thermometer based on gastric acid battery.

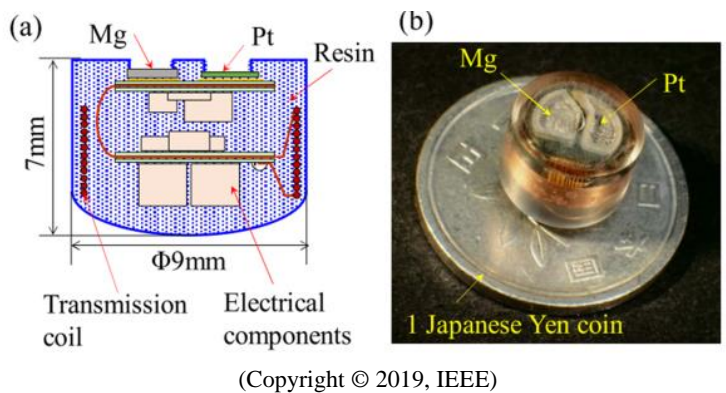

Fig. 2. Previous device utilizing a folded rigid flexible PCB and a separate coil. (a) Cross-sectional schematic. (b) Picture of the device [33].

wearable sensor technologies [1][2][3]. In the future, minimally invasive and safer technologies should be developed as next-generation healthcare technology [4][5].

For example, ingestible sensors, which can be swallowed by a user to monitor vital data in the body, will become a promising technology in the near future [6][7][8]. Thus far, various ingestible sensors have been developed to measure core body temperature [9][10][11], gas [12], microbiome [13], $\mathrm{pH}$ [14], pressure [15] and capsule-type imagers [16]. Among them, it is predicted that ingestible thermometers will produce the largest market with a steady growth rate [17]. One reason for this prediction is that core body temperature and its temporal patterns, especially during sleep, are fundamental vital signs, and thus can be used for various purposes. Hence, their 


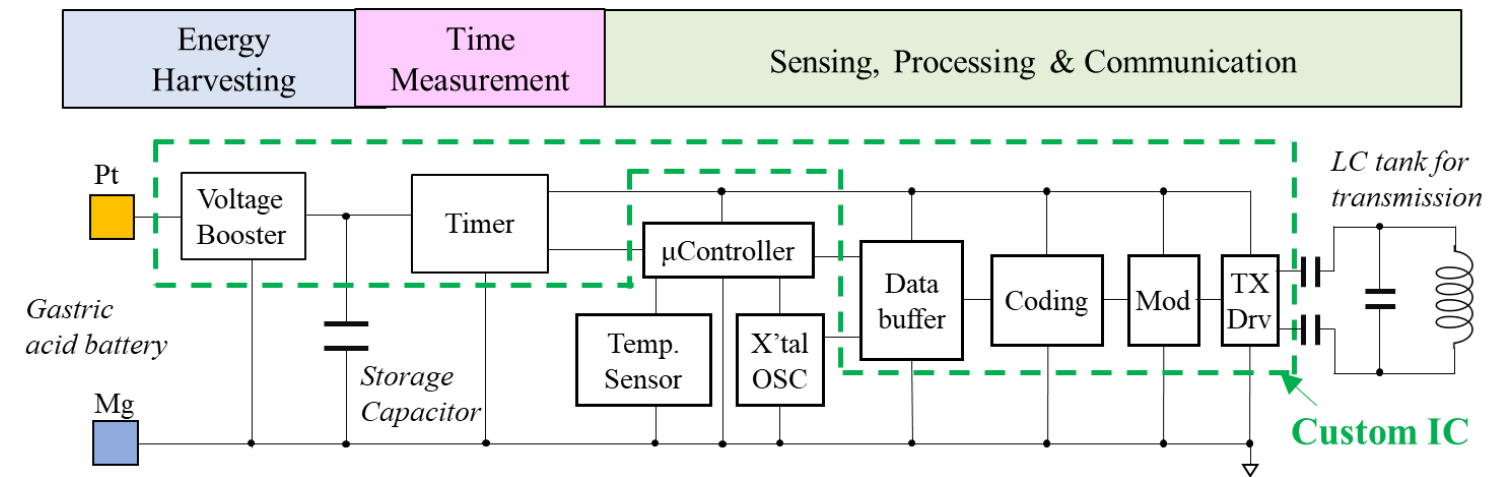

Fig. 3. System outline of the ingestible thermometer based on gastric acid power generation. The parts inside the dashed green line were implemented in a custom IC.

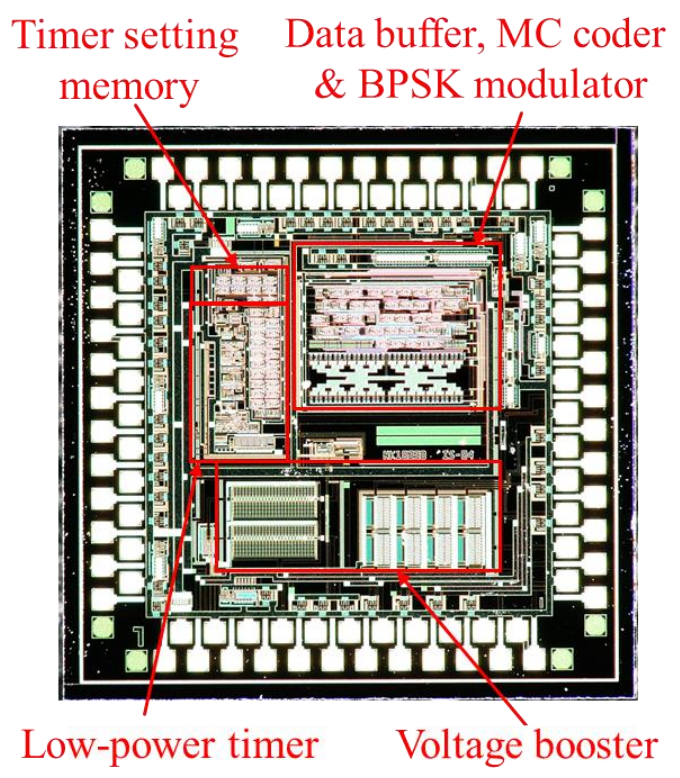

Fig. 4. Layout schematic of the custom IC $(0.5 \mu \mathrm{m} / 5 \mathrm{~V}$ CMOS process). The chip size is $1.9 \mathrm{~mm} \times 1.9 \mathrm{~mm}$.

monitoring and logging can potentially help to detect various diseases earlier or more accurately, such as menstrual disorders [18], polycystic-ovary syndrome [19], depression [20], infection [21], and a specific sleep disorder [22][23]. In addition, the core body-temperature rhythm is a strong indicator of the circadian rhythm (inner-body clock). In particular, it is important to observe the timing of the lowest body temperature during sleep because it is an indicator of the phase of the rhythm. An abnormal phase shift from the sleep-wake rhythm and/or social clock increases the risk of disordered sleep, cancer, obesity, depression, diabetes, infertility, etc. [24][25][26][27]. Mental and athletic performance also strongly depends on the rhythm [28][29]. Thus, aggressive monitoring and calibration contribute not only to disease therapy and diagnosis, but also to empowerment. An attractive advantage of the ingestible thermometer is that it can obtain reliable data more robustly to the environmental or contact conditions compared with other non-contact or contact-type sensors. It has been proven that it is possible to accurately measure body temperature, which is strongly correlated with rectal temperature [21][30].

Ingestible thermometers are already commercially available. However, these sensors have a critical problem: they use button batteries as energy sources. Hence, these existing devices have a risk of causing severe injuries, such as damage to the gastrointestinal tract due to the discharge current or toxic electrolyte if the button battery is exposed inside the body. Issues of disposability and self-discharge also arise with the fabrication of devices using primary batteries. Wireless power from the outside of the body to an ingested device has been proposed [9]. However, difficulty may arise in efficiently supplying power to a device whose position is unknown in the body. Consequently, large exposure amounts of electromagnetic radiation to the body may be required to supply sufficient power, which poses further health risks. In addition, these devices are too expensive for consumer use. These problems may limit the use of the device in specialized applications, such as in athletics or the military.

Thus, in previous studies, we developed a core-body thermometer based on gastric acid power generation even after the device has passed through the stomach, as shown in Fig. 1 [31][32][33]. The gastric acid power generation charges electrical energy into a storage capacitor, which is safer than a button battery, in the device. This concept reduces the environmental burden of disposing of the device after use. Since the device is spontaneously charged when taken, there is no need to worry about deterioration like button batteries. Thus, the device has quality robustness to product design, and the restrictions of logistics and storage conditions are minimized.

The idea of using gastric acid power generation has been considered for a long time. Medication management devices are a recent example [34][35]. An ingestible thermometer that is meant to remain within the stomach for a long time has been reported [36]. However, these devices are assumed to be operated only in the stomach because the power generation virtually stops in the bowels. The average residence time in the stomach is approximately $1 \mathrm{~h}$. Thus, the designs of these systems do not meet our intended application, that is, observation of temporal patterns of core body temperature for ten or more hours. In a previous study, we successfully prototyped a tablet-shaped device and demonstrated in vivo 
telemetry by letting a dog swallow the device [33]. However, the device was fabricated by folding a rigid-flexible circuit board, as shown in Fig. 2. The coil antenna was mounted manually. These complicated assembly processes make automatic fabrication difficult and prevent the reduction of the fabrication cost. Our goal is to supply a device for various applications in consumer use by cost-effective mass production. Moreover, long-time operation, such as more than $10 \mathrm{~h}$, has not yet been demonstrated. Thus, our concept has not yet been completely proven.

Therefore, in this study, we designed a suitable structure for an ingestible thermometer with superior mass-production, and then prototyped the device. We then demonstrated in vivo telemetry for a long time.

\section{SYSTEM DESIGN}

Fig. 3 depicts a system diagram of the ingestible thermometer proposed in this study. When the device is ingested, power generation starts by bringing the electrodes (e.g., Mg and Pt electrodes) into contact with a gastric acid fluid, and then drives a self-excited-oscillating circuit for voltage boosting. Subsequently, a clock pulse drives a voltage boost circuit, and the electric energy is charged in a multilayer ceramic capacitor (MLCC) at the boosted voltage. An MLCC was chosen as a storage capacitor because it is inexpensive, easy to procure, safe for the body because of the absence of a toxic electrolytic solution, and can be charged quickly. The charged energy becomes an energy source for this system after it passes through the stomach. The timer circuit manages the operation timing of the subsequent elements, such as temperature sensing and telecommunication functions. When a preset timing arrives, the charged energy is supplied to them. A general-purpose microcontroller obtains temperature data from a temperature sensor through an inter-integrated circuit interface. The microcontroller then creates a data packet and sends it to a data buffer. The data are bit-rate converted, encoded, modulated, and eventually sent to an external receiver via magnetic-field coupling communication. This communication method does not require adhesives or direct contact between the receiver and skin to receive data from the ingested device. In this study, an $11.3-\mathrm{MHz}$ carrier frequency generated by a crystal oscillator was used because such a relatively low radio-frequency carrier should have a well-balanced performance between biological permeability and efficiency as a limited-size transmission antenna in the ingestible device. This contributes to ensuring safety in the human body and reducing energy consumption in telecommunications. To minimize the driving energy loss, an LC parallel resonant circuit, which is driven by differential outputs, is used to generate a modulated magnetic field. Manchester encoding and binary phase-shift keying (BPSK) were adopted as the encoding and modulating methods, respectively. The content of the data packet is described in our previous study [32].

Some functional elements were implemented in a custom integrated circuit (IC) (inside the dashed green line in Fig. 3). This contributes not only to miniaturization of the device, but

\section{(1) Prepare PCBs mounted with electrical components}
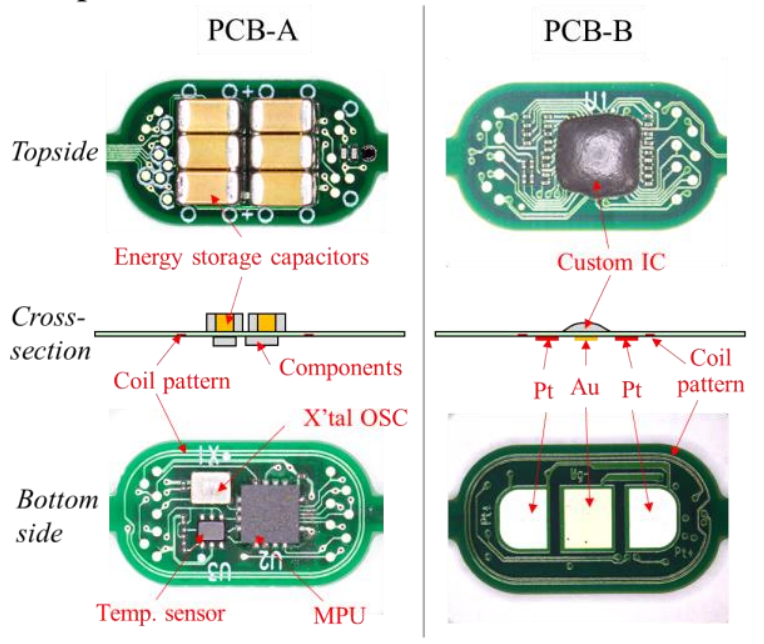

(2) Stack PCBs and electrically connect with solder ball

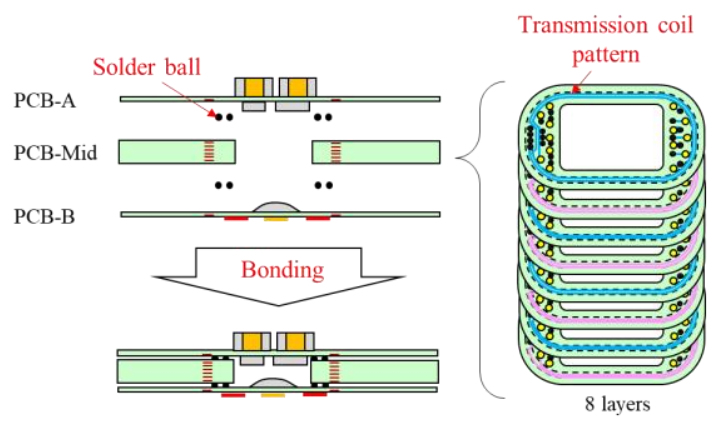

\section{(3) 1. Cut out devices, 2. mount Mg plate, and 3. package with a resin}

1.
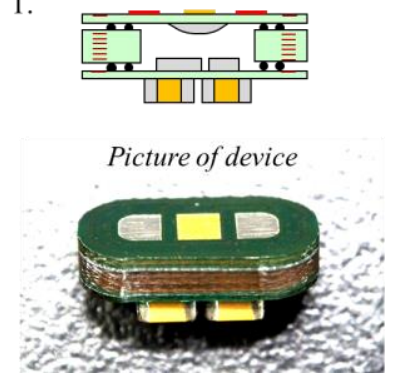

2.

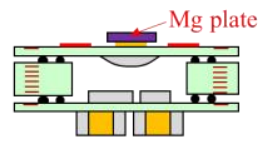

3.

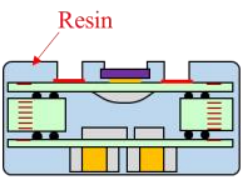

Fig. 5. Fabrication process of the ingestible thermometer utilizing staking of PCBs with coil patterns.

also to reducing the energy consumption for the encoding and modulating processes. Fig. 4 illustrates a schematic layout of the custom IC. The die was a square with a side length of 1.8 $\mathrm{mm}$. A conventional 0.5- $\mu \mathrm{m}$ CMOS process was employed for cost reduction and to ensure a wide range of operating voltages. The circuit design of the voltage booster is described in [37]. The chargeable energy in an MLCC is several orders of magnitude lower than that of general button batteries. Thus, energy saving is required for not only the active mode 

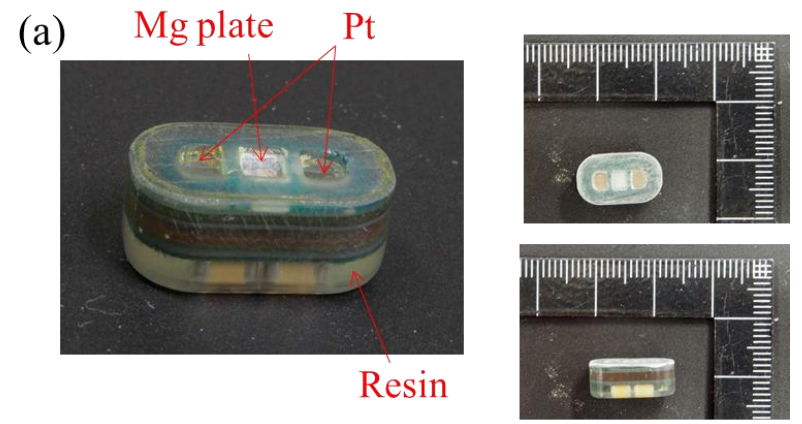

(b) Transmission coil pattern

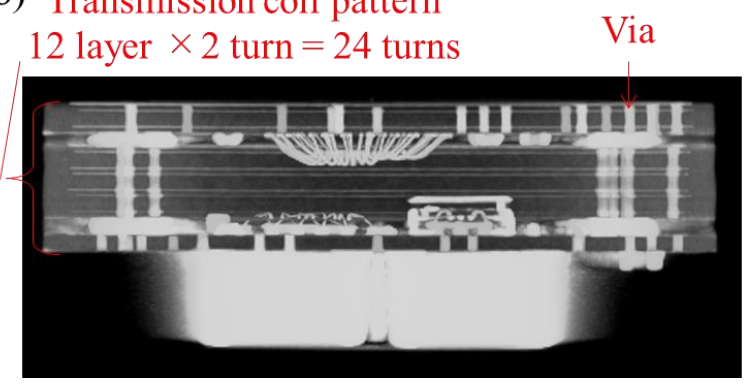

Fig. 6. (a) Picture of the prototyped device. (b) Cross-sectional view observed by X-ray computed tomography.

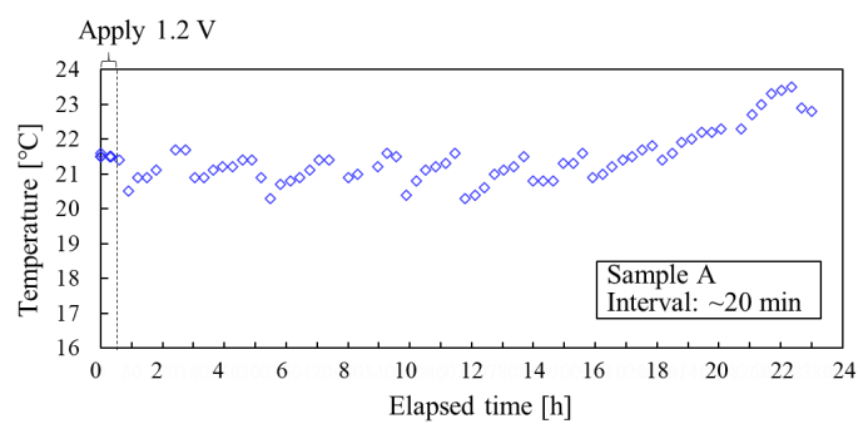

Apply $1.2 \mathrm{~V}$

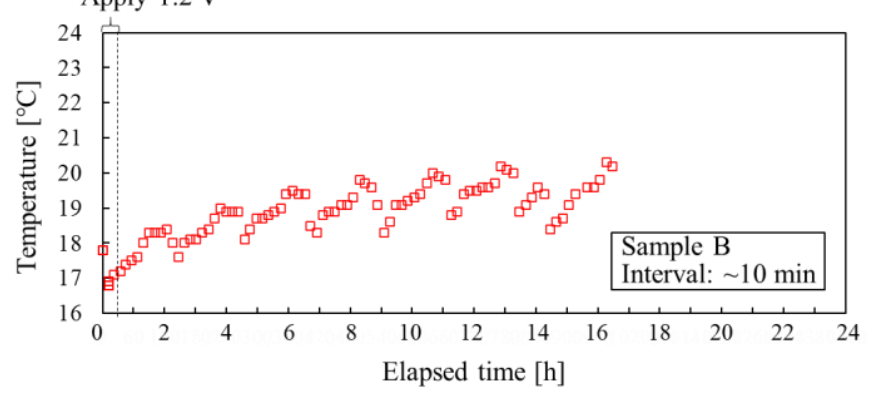

Fig. 7. Operation test results of the devices in vitro. The measurement cycles of sample A and sample B are $20 \mathrm{~min}$ and $10 \mathrm{~min}$, respectively.

TABLE I

PHYSICAL AND TECHNICAL CHARACTERISTICS OF THE INGESTIBLE THERMOMETERS [38].

\begin{tabular}{|c|c|c|c|c|c|}
\hline $\begin{array}{c}\text { Name } \\
\text { (Developer) }\end{array}$ & $\begin{array}{l}\text { CorTemp } \\
\text { (HQ. Inc.) }\end{array}$ & $\begin{array}{c}\text { e-Celsius } \\
\text { (BodyCap) }\end{array}$ & $\begin{array}{c}\text { myTemp } \\
\text { (myTemp BV) }\end{array}$ & $\begin{array}{c}\text { VitalSense } \\
\text { (Royal Philips) }\end{array}$ & This work \\
\hline Dimensions & $\begin{array}{l}10.9 \mathrm{~mm}(\Phi) \\
\times \\
22.4 \mathrm{~mm}(\mathrm{~L})\end{array}$ & $\begin{array}{c}8.9 \mathrm{~mm}(\Phi) \\
\times \\
17.7 \mathrm{~mm}(\mathrm{~L})\end{array}$ & $\begin{array}{c}8.0 \mathrm{~mm}(\Phi) \\
\times \\
20.0 \mathrm{~mm}(\mathrm{~L})\end{array}$ & $\begin{array}{c}8.7 \mathrm{~mm}(\Phi) \\
\times \\
23.0 \mathrm{~mm}(\mathrm{~L})\end{array}$ & $\begin{array}{c}8.3 \mathrm{~mm}(\mathrm{~W}) \\
\times \\
6.0 \mathrm{~mm}(\mathrm{H}) \\
\times \\
14.3 \mathrm{~mm}(\mathrm{~L})\end{array}$ \\
\hline Weight & $2.8 \mathrm{~g}$ & $1.7 \mathrm{~g}$ & $1.3 \mathrm{~g}$ & $1.5 \mathrm{~g}$ & $1.3 \mathrm{~g}$ \\
\hline Power source & $\begin{array}{l}\text { Silver oxide } \\
\text { battery }\end{array}$ & $\begin{array}{l}\text { Silver oxide } \\
\text { battery }\end{array}$ & $\begin{array}{l}\text { Extracorporeal } \\
\text { wireless power } \\
\text { transfer }\end{array}$ & $\begin{array}{l}\text { Silver oxide } \\
\text { battery }\end{array}$ & $\begin{array}{c}\text { Gastric acid } \\
\text { battery }\end{array}$ \\
\hline
\end{tabular}

(temperature sensing and telecommunication, etc.), but also for the non-active mode. Thus, an ultralow-power timer circuit was implemented in this custom IC. The power consumption was as small as $\sim 7.5 \mathrm{nA}$ at $4 \mathrm{~V}$. The period of the timer in the custom IC can be set by the timer set memory, which is written by the microcontroller.

\section{FABRICATION OF DEVICE}

Fig. 5 illustrates the fabrication flow of the device. A coil pattern was formed on the outer periphery of the components. First, on PCB-B, a Pt thin film was electrically plated on the Au pads as a positive electrode of the gastric acid battery in advance. All electronic components, except the custom IC, were then mounted on rigid PCBs (PCB-A and PCB-B), and the bare chip of the custom IC was mounted with wire bonding, as shown in Fig. 5(1). In this study, PIC16LF1503 (Microchip Technology Inc.), TMP112 (Texas Instruments Inc.), and
KC2016K (Kyocera Corp.) were used as the microcontroller, temperature sensor, and crystal oscillator, respectively. The accuracy and resolution of the temperature sensor were $0.5^{\circ} \mathrm{C}$ and $0.0625{ }^{\circ} \mathrm{C}$, respectively. The PCBs were stacked and electrically connected via an intermediate PCB (labeled "PCB-Mid") by a soldering reflow process, as shown in Fig. 5(2). PCB-Mid not only had an embedded multilayer coil pattern, but also prevented the interference of components on PCB-A and PCB-B. The device was then cut out, as shown in Fig 5(3). Then, a 250- $\mu$ m-thick Mg plate was bonded via an Ag paste. Finally, the PCBs were packaged with epoxy resin. Only the $\mathrm{Mg}-\mathrm{Pt}$ electrodes are exposed for contact with gastric acid.

Fig. 6 (a) depicts images of the prototype device. The dimensions were 14.3 (length) $\times 8.3$ (width) $\times 6.0$ (height) $\mathrm{mm}$, which are smaller than other existing ingestible thermometers, as listed in Table I. Embedding a coil antenna in a PCB and eliminating a button battery considerably contributed to miniaturization. The size reduction contributes to reducing the 


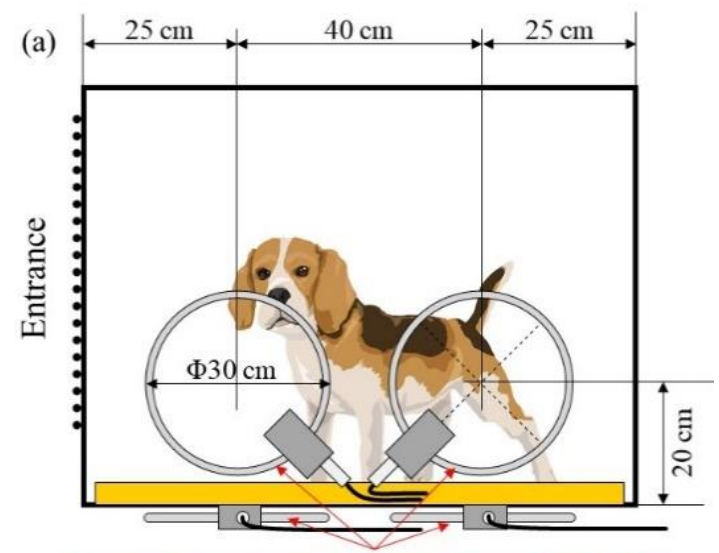

Loop antennas set on the bottom and both sides.

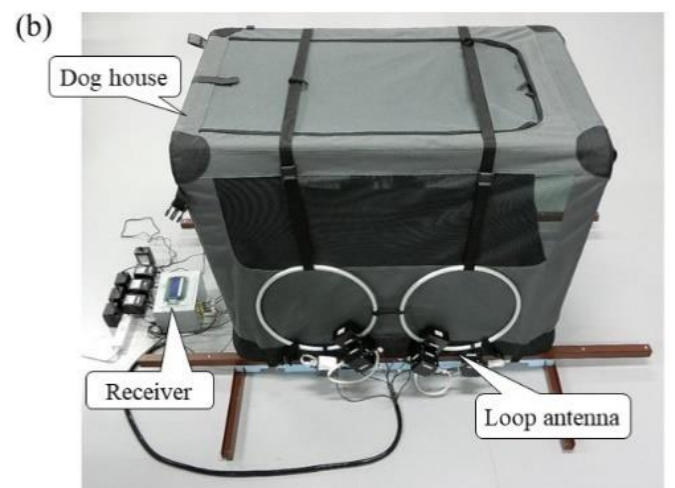

Fig. 8. (a) Schematic illustration of the layout of the receiver antennas for the in vitro telemetry with a dog. (b) Picture of the actual setup.

risk of leaving the device in the body and blocking the digestive tract [38]. The X-ray computed tomography image (Fig. 6(b)) depicts the coil antennas on each layer, which are connected vertically with the vias in the device. The number of turns was 24 , and the inductance and resistance were approximately $6 \mu \mathrm{H}$ and $14 \Omega$, respectively.

\section{IN VITRO OPERATION TEST}

An operation test of the prototype devices was performed in vitro as a preliminary experiment. In this experiment, metal probes were placed on the gastric-acid-battery electrodes of $\mathrm{Mg}$ and Pt. Then, $1.2 \mathrm{~V}$ was applied for $30 \mathrm{~min}$ assuming gastric-acid power generation occurs in the stomach. This applied voltage was increased to approximately $4.2 \mathrm{~V}$ by the voltage booster circuit; thus, the MLCC was charged at this voltage. We then stopped the voltage application and observed the performance of the device by using the charged energy, assuming the device moved to the bowels. The data signals transmitted from the device were received by a commercially available magnetic loop antenna (LA400, AOR Ltd). The distance between the device and antenna was estimated to be approximately $20 \mathrm{~cm}$. This experiment was performed under ambient conditions using a general air conditioner. We prepared two samples with a timer circuit interval of approximately $20 \mathrm{~min}$ (sample A) and $10 \mathrm{~min}$ (sample B). The measurement frequency of the latter device was doubled. In other words, the accumulated energy consumption for the active mode was twice that of the former.

Fig. 7 illustrates the variation of the measured temperature in time in the operation test. The temperature change of the experimental room according to the air-conditioner was monitored. Sample A operated for approximately 23 hours while over 16 hours was achieved in sample B as a measurement time. Note that the operation experiments for each sample were conducted on different days. Therefore, the values of the measured room temperatures are different largely.

The existing ingestible thermometers can monitor the temperature in a dozen-second period for a few days. In contrast, the current design of our device measured in periods of the order of minutes for ten or more hours because the available energy of the device using an MLCC is several orders of magnitude smaller than that of a typical button battery. Because the energy charged in the MLCC is limited, there is a trade-off between the maximum measurable time and the number of measurements. However, various applications, such as measurement of basal temperature during sleep, circadian rhythm, and bed-side monitoring of infection patients, probably do not require frequent monitoring. Thus, our device and existing devices are expected to be used differently. In addition, we can expect that advancements in the energy density of MLCCs will further increase the maximum measurable time or measurement frequency in the future.

\section{Demonstration of IN VIVO LONG-Time TELEMETRY}

Finally, an in vivo telemetry experiment was performed by ingesting the device in a male beagle dog. We first prepared a dog house surrounded by receiver antennas for this experiment, as shown in Fig. 8. A total of six loop antennas were attached; two antennas were each placed on the bottom and the near/far sides. The devices (samples A and B) were then swallowed by the dog. In addition, CorTemp ${ }^{\mathrm{TM}}$ (supplied by the HQ. Inc) was used as a reference. Afterward, the dog was put to sleep using a sedating agent (secobarbital sodium), and the dog was put in the dog house. The rectal temperature was also monitored by inserting a thermal probe (CTM-303, Terumo Corporation) during the sleeping state. After approximately $30 \mathrm{~min}$, the rectal thermal probe was removed, and the dog was removed from the dog house and placed on a medical table. The inside of the stomach was observed using an endoscope. Afterward, the dog was put back in the dog house, and the rectal probe was set until the dog became awake during the telemetry. The rectal probe was then removed before the dog became completely awake, and the core-body temperature was monitored using only the ingestible devices for $24 \mathrm{~h}$. The devices stayed in the body and were not excreted during this experiment of 24 hours.

All experimental procedures conformed to "the Act on Welfare and Management of Animals" and "Regulations for Animal Experiments at Ina Research Inc.," and they were reviewed by the Institutional Animal Care and Use Committee of Ina Research Inc. (Protocol number: 21016, Approval date: 

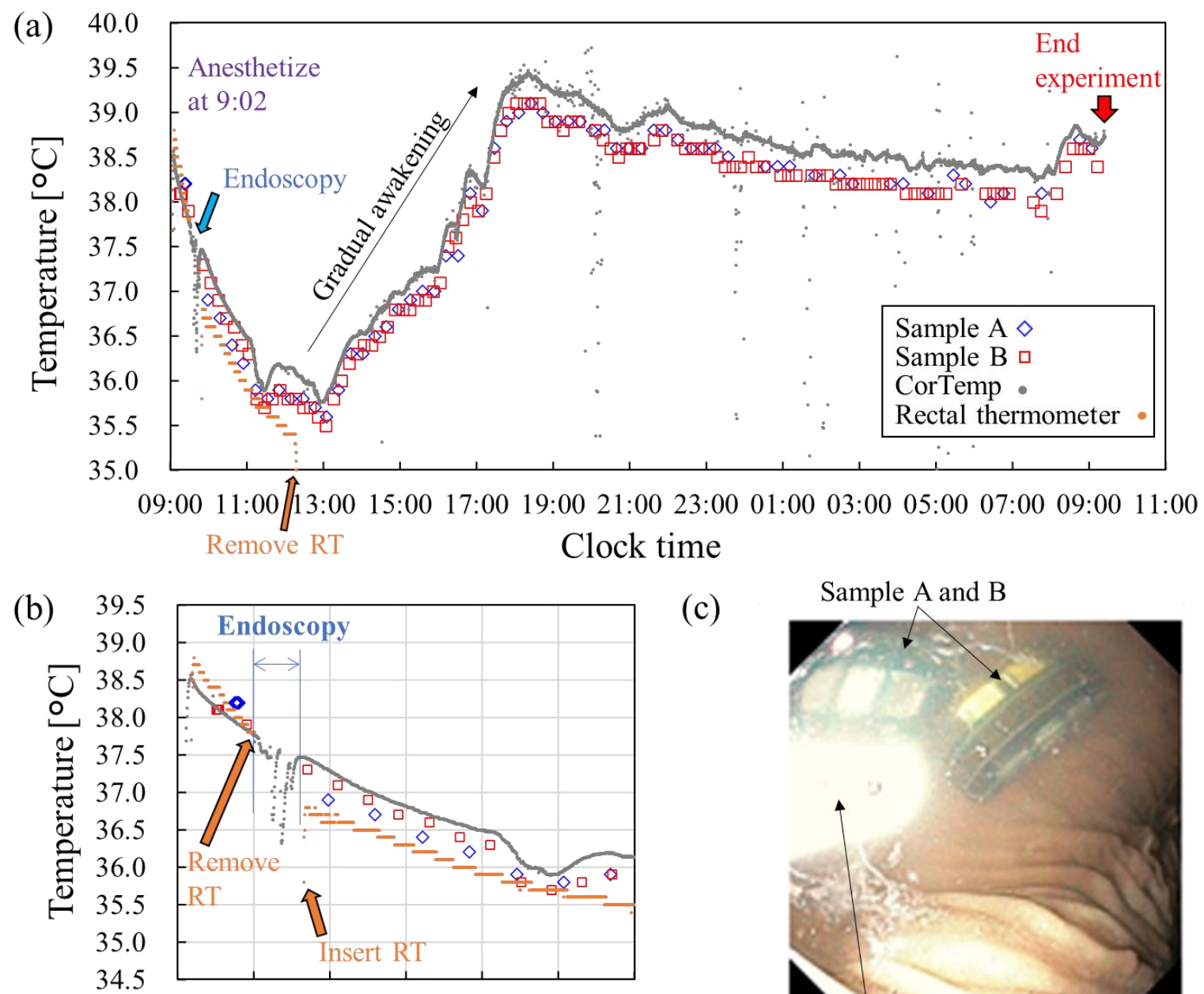

09:00 09:30 10:00 10:30 11:00 11:30 12:00 Clock time

(c)

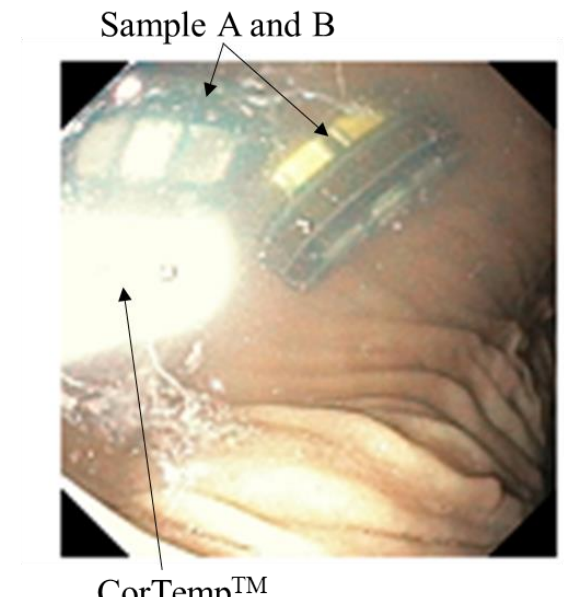

Fig. 9. Demonstration of the in vivo telemetry experiment. (a) Raw temperature data recorded for 24 hours. RT means rectal thermometer. (b) Detailed plot of the recorded temperature data under sedation. (c) Endoscopic image of the devices in the stomach.

January 20, 2021)

Fig. 9 illustrates the temperature recorded in the measurement experiment. As seen in Fig. 9 (a), the long-time measurement for $24 \mathrm{~h}$ was demonstrated successfully. The operation time of "sample B" was longer than that of the in vitro test results. The device probably stayed and charged in the stomach for a longer time than expected. For at least $30 \mathrm{~min}$ after ingestion, all devices were observed in the stomach, as shown in Fig. 9(c). In addition, power generation from the galvanic effect of the Mg-Pt electrode may have occurred even in the intestine, although the amount is much smaller than in the stomach. This small amount of power generation might have extended the operating time.

The temperature variations of our devices, CorTemp ${ }^{\mathrm{TM}}$, and the rectal thermometer were almost parallel. The temperature of CorTemp ${ }^{\mathrm{TM}}$ always appears to be $0.2^{\circ} \mathrm{C}-0.3^{\circ} \mathrm{C}$ higher than that of our devices. This might be due to the differences in the sensing accuracy among the devices. Alternatively, CorTemp ${ }^{\mathrm{TM}}$ might have been slightly heated up because of its high-frequency measurement of a few tens of seconds. As seen in Fig. 9 (b), the variation in the rectal temperature shifted before and after the endoscopic observation. The rectal thermometer was then removed for observation. This might indicate that even the rectal temperature was influenced by the contact condition between the inserted probe and the rectal wall or feces. In this experiment, the rectal temperature almost matched the data measured with our devices before the endoscopic observation, and it was $0.2{ }^{\circ} \mathrm{C}-0.3{ }^{\circ} \mathrm{C}$ lower after the observation. This suggests that the ingestible thermometer has the potential to provide a more robust sensing method for the core body temperature.

Therefore, we successfully developed a fabrication process that makes it easy to integrate a coil antenna into an ingestible thermometer. We demonstrated that this thermometer based on gastric acid power generation can measure the core body temperature of a living animal for a long time.

\section{CONCLUSION}

In this study, we prototyped an ingestible thermometer with a built-in coil antenna, whose operation energy is charged by a 
gastric acid battery. The custom IC implemented with essential functions, such as ultralow-power timer, coding, modulation, and transmission driver circuits, was prepared to realize the conceptual device. We also developed an implementation process for a coil antenna in the device by vertically stacking PCBs with loop patterns. Consequently, the integration and assembly process of all components were considerably simplified compared with the previous method of using rigid-flexible circuit boards [32][33]. The dimensions of the fabricated device were $14.3 \mathrm{~mm}$ (length) $\times 8.3 \mathrm{~mm}$ (width) $\times$ $6.0 \mathrm{~mm}$ (height). This size is smaller than that of other existing ingestible thermometers with general button batteries. Finally, we successfully demonstrated in vivo telemetry for $24 \mathrm{~h}$ in cycles of approximately 10 or $20 \mathrm{~min}$. The temperature variations in time between our devices and reference sensors were almost parallel. This performance has prospects for easily and robustly measuring basal body temperature during sleep, the circadian rhythm, and fever type. In addition, the resolution and accuracy of the device will be further improved by adopting a higher-temperature IC in the system. Our device has the potential to become a safe and inexpensive ingestible thermometer that would be more widely used than existing products. We believe that our ingestible thermometer is the first step toward creating an ingestible sensor available in daily life for preventive medicine and health promotion.

\section{REFERENCES}

[1] J. Tu et al., "The era of digital health: A review of portable and wearable affinity biosensors," Adv. Funct. Mater., vol. 30, no. 29, pp. 1-30, 2020.

[2] H. Zhang et al., "MHealth technologies towards Parkinson's disease detection and monitoring in daily life: A comprehensive review," IEEE Rev. Biomed. Eng., vol. 14, pp. 71-81, 2021.

[3] F. Yang et al., "Internet of things enabled data fusion method for sleep healthcare applications," IEEE Internet Things J., vol. 7, no. 4, pp. 1-1, 2021

[4] S. Carrara, "Body dust: Well beyond wearable and implantable sensors," IEEE Sens. J., in press, pp. 1-1, 2020.

[5] H. Dinis and P. M. Mendes, "A comprehensive review of powering methods used in state-of-the-art miniaturized implantable electronic devices," Biosens. Bioelectron., vol. 172, no. October 2020, p. 112781, 2021.

[6] C. Steiger et al., "Ingestible electronics for diagnostics and therapy," Nat. Rev. Mater., vol. 4, no. 2, pp. 83-98, 2019.

[7] L. A. Beardslee et al., "Ingestible sensors and sensing systems for minimally invasive diagnosis and monitoring: The next frontier in minimally invasive screening," ACS Sensors, vol. 5, no. 4, pp. 891-910, 2020 .

[8] A. S. Sharova et al., "Edible electronics: The vision and the challenge," Adv. Mater. Technol., vol. 6, no. 2, 2021.

[9] C. C. W. G. Bongers et al., "Validity and reliability of the myTemp ingestible temperature capsule," J. Sci. Med. Sport, vol. 21, no. 3, pp. 322-326, 2018

[10] C. C. W. G. Bongers et al., "Validity, reliability, and inertia of four different temperature capsule systems," Med. Sci. Sports Exerc., vol. 50, no. 1, pp. 169-175, 2018.
[11] C. O'Brien et al., "Telemetry pill measurement of core temperature in humans during active heating and cooling," Med. Sci. Sports Exerc., vol. 30, no. 3, pp. 468-472, 1998.

[12] K. Kalantar-Zadeh et al., "A human pilot trial of ingestible electronic capsules capable of sensing different gases in the gut," Nat. Electron., vol. 1, no. 1, pp. 79-87, 2018.

[13] C. Zhu et al., "A packaged ingestible bio-pill with 15-pixel multiplexed fluorescence nucleic-acid sensor and bi-directional wireless interface for in-vivo bio-molecular sensing," in 2020 IEEE Symposium on VLSI Circuits, 2020, vol. 10, no. 3, pp. 1-2.

[14] Heidelberg Medical, Gastric pH Diagnostic System and pH Capsule. Available: https://www.phcapsule.com/

[15] C. H. Liao et al., "An ingestible electronics for continuous and real-time intrababdominal pressure monitoring," J. Pers. Med., vol. 11, no. 1, pp. 110,2021

[16] T. Nakamura and A. Terano, "Capsule endoscopy: Past, present, and future," J. Gastroenterol., vol. 43, no. 2, pp. 93-99, 2008.

[17] Ingestible Sensors Market Size, Share \& Trends Analysis Report By Vertical (Sports, Medical), By Component (Sensors, Software), By Sensor Type (Temperature, Pressure, Image Sensors), And Segment Forecasts, 2018 - 2024. Grand View Research, Inc, Jul, 2018, Report ID: 978-1-68038-923-4.

[18] A. Palmer, "Basal body temperature determinations in the management of menstrual disorders." Clin Obstet Gynecol. vol. 2, no 1, pp. 153-79. 1959

[19] W. W. Webster and B. Smarr, "Using circadian rhythm patterns of continuous core body temperature to improve fertility and pregnancy planning," J. Circadian Rhythms, vol. 18, no. 1, pp. 1-8, 2020.

[20] B. P. Hasler et al., "Phase relationships between core body temperature, melatonin, and sleep are associated with depression severity: Further evidence for circadian misalignment in non-seasonal depression," Psychiatry Res., vol. 178, no. 1, pp. 205-207, 2010.

[21] F. Huang et al., "Ingestible sensors correlate closely with peripheral temperature measurements in febrile patients," J. Infect., vol. 80, no. 2, pp. 161-166, Feb. 2020.

[22] M. Morris et al., "Sleep-onset insomniacs have delayed temperature rhythms," Sleep, vol. 13, no. 1, pp. 1-14, Jan. 1990.

[23] L. C. Lack et al. "The relationship between insomnia and body temperatures," Sleep Med. Rev., vol. 12, no. 4, pp. 307-317, Aug. 2008.

[24] S. Crnko et al., "Circadian rhythms and the molecular clock in cardiovascular biology and disease," Nat. Rev. Cardiol., vol. 16, no. 7, pp. 437-447, 2019.

[25] Y. Leng et al., "Association between circadian rhythms and neurodegenerative diseases," Lancet Neurol., vol. 18, no. 3, pp. 307-318, 2019.

[26] M. Gombert et al., "The connection of circadian rhythm to inflammatory bowel disease," Transl. Res., vol. 206, pp. 107-118, 2019.

[27] S. Kaur et al., "Circadian rhythm and its association with birth and infant outcomes: Research protocol of a prospective cohort study," BMC Pregnancy Childbirth, vol. 20, no. 1, pp. 1-11, 2020.

[28] N. Goel et al., "Circadian rhythms, sleep deprivation, and human performance," Prog. Mol. Biol. Transl. Sci., vol. 119, pp. 155-190, 2013.

[29] C. E. Kline et al., "Circadian variation in swim performance," J. Appl. Physiol., vol. 102, no. 2, pp. 641-649, Feb. 2007.

[30] B. Edwards et al., "A comparison of the suitabilities of rectal, gut, and insulated axilla temperatures for measurement of the circadian rhythm of core temperature in field studies," Chronobiol. Int., vol. 19, no. 3, pp. 579-597, Jan. 2002. 
[31] S. Yoshida et al., "Concept proof of low-energy consumption and compact ingestible thermometer based on gastric acid power generation," IEEJ Trans. Sensors Micromachines, vol. 138, no. 4, pp. 164-169, 2018.

[32] S. Yoshida et al., "Development of tablet-shaped ingestible core-body thermometer powered by gastric acid battery," IEEE Sens. J., vol. 18, no. 23, pp. 9755-9762, Dec. 2018.

[33] S. Yoshida et al., "Proof of concept for tablet-shaped ingestible core-body thermometer with gastric acid battery," in 2019 IEEE 1st Global Conference on Life Sciences and Technologies (LifeTech), 2019, pp. 182184.

[34] H. Hafezi et al., "An ingestible sensor for measuring medication adherence," IEEE Trans. Biomed. Eng., vol. 62, no. 1, pp. 99-109, 2015.

[35] G. P. Flores et al., "Performance, reliability, usability, and safety of the id-cap system for ingestion event monitoring in healthy volunteers: A pilot study," Innov. Clin. Neurosci., vol. 13, no. 9-10, pp. 12-19, 2016.
[36] P. Nadeau et al., "Prolonged energy harvesting for ingestible devices," Nat. Publ. Gr., vol. 1, no. February, pp. 1-8, 2017.

[37] S. Yoshida et al., "Prototyping of an sll-pMOS-based cross-coupled voltage multiplier in single-well CMOS technology for energy harvesting utilizing a gastric acid battery," Electronics, vol. 8, no. 7, p. 804, Jul. 2019.

[38] C. Steiger et al., "Ingestible electronics for diagnostics and therapy," Nat. Rev. Mater., vol. 4, no. 2, pp. 83-98, 2019.

[39] C. C. W. G. Bongers et al., "Validity, reliability, and inertia of four different temperature capsule systems," Med. Sci. Sports Exerc., vol. 50, no. 1, pp. 169-175, 2018. 